\title{
STUDIES ON THE PATHOGENESIS OF THALASSAEMIA
}

\author{
BY \\ GIOVANNI ASTALDI AND PAOLO TOLENTINO \\ From the Departments of Internal Medicine, University of Pavia, and of Paediatrics, \\ University of Genoa, Italy
}

(RECEIVED FOR PUBLICATION SEPTEMBER 27, 1951)

This short paper is a study of erythrocyte production and destruction in thalassaemia, undertaken so as to throw light on the pathogenesis of the disease. Thalassaemic bone marrow has been cultured in vitro (Astaldi, Tolentino, and Sacchetti, 1951a, b, c) thereby allowing the separate study of the intensity of differentiation and the proliferation of erythroblasts. By the culture method it is possible to visualize these activities more exactly than by static morphological criteria such as the myelogram, the maturation curve, the mitotic rate, and the karyokinetic histogram.

\section{Method}

Differentiation.-Differentiation was studied by keeping the surviving medullary cells in a Carrel flask containing a liquid medium consisting of plasma and Tyrode's solution (Astaldi and Tolentino, 1949). At the beginning of the experiment, and then at six to 12-hour intervals, a count was made in absolute numbers per c.mm. of the erythroblasts and reticulocytes (at different stages of maturation) surviving in the culture.

As under these conditions erythropoiesis quickly fades while differentiation continues, the total number of erythroblasts gradually decreases and may ultimately disappear. The first to diminish in numbers are the basophilic erythroblasts followed by the polychromatic and finally by the orthochromatic erythroblasts. The reticulocytes, too, disappear as they ripen to adult erythrocytes.

Proliferation.-Proliferation was studied by explanting fragments of bone marrow on to solid medium containing colchicine at an optimum concentration (Astaldi and Mauri, 1949, 1950). Owing to the "stathmokinetic" action of the colchicine, further development of the cells entering mitosis is inhibited at pre-metaphase, the result being that the number of mitoses in the culture (the "stathmokinetic rate") gradually increases with time. The rapidity of proliferation can thus be deduced from the increase in the number of mitoses reached in a given time.

\section{Results}

Proliferation.-The mitotic activity of the basophilic erythroblast was found in all the three forms of thalassaemia (major, minor, and minima) to be far greater than that of the basophilic erythroblasts of a normal subject. In thalassaemia major and minor the rate is constantly high ; in thalassaemia minima it is more variable (Fig. 1). On the other hand, the proliferation activity of the polychromatic erythroblasts is no greater than normal in all three forms of thalassaemia. The orthochromatic erythroblasts do not divide.

Differentiation.-It is possible that in all three forms of thalassaemia there is some delay in the development of the polychromatic from the basophilic erythro- 


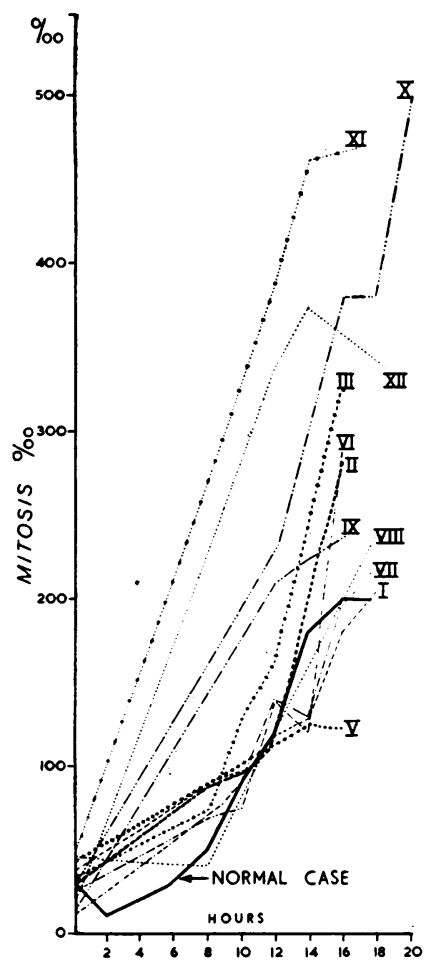

(a)

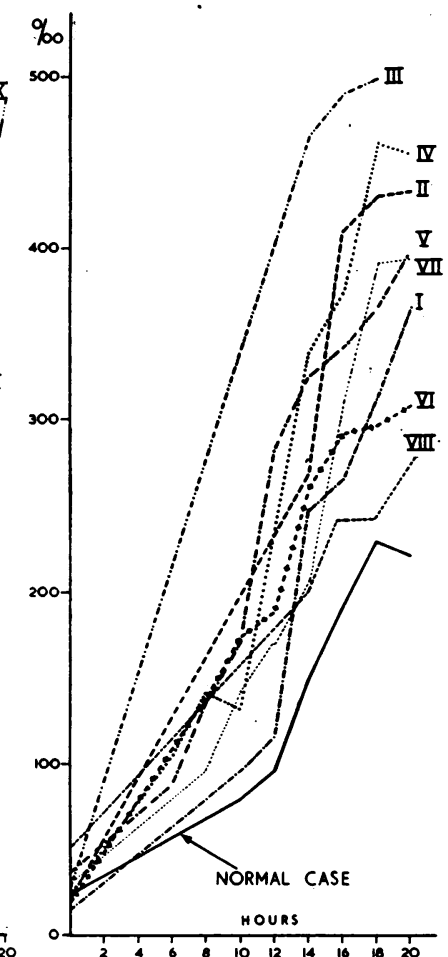

(b)

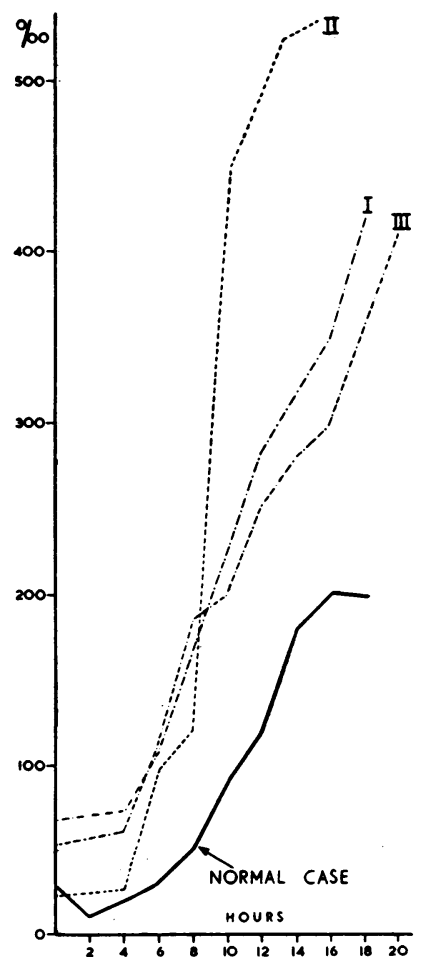

(c)

FIG. 1.-(a) Thalassaemia minima; (b) thalassaemia major; (c) thalassaemia minor.

blast. In the most serious cases of thalassaemia major the differentiation of orthochromatic erythroblasts into erythrocytes is retarded (delayed enucleation), this phenomenon being easier to observe because the erythroblasts do not proliferate at the orthochromatic stage (Fig. 2). Such a phenomenon is, however, not exclusively associated with thalassaemia ; it has been observed in other secondary erythroblastic anaemias associated with hyperhaemolysis.

The medullary erythroblastosis which is found in all three forms of thalassaemia can be explained by an increased formation of erythroblasts from the histoid matrix accompanied by increased proliferation of the basophilic erythroblasts. In the worst cases this may be associated with a slowing down in the differentiation of the orthochromatic erythroblasts.

"Crossed" Plasma Experiments.-Experiments have been carried out using a " crossed " plasma technique, thalassaemic marrow being cultured in normal plasma and vice versa. It has been observed that thalassaemic basophilic erythroblasts proliferate in normal plasma and in thalassaemic plasma at the same degree of intensity (Fig. 3); also that the proliferation of normal erythroblasts does not increase in thalassaemic plasma (Fig. 3), and that the differentiation of thalassaemic erythroblasts follows the same course in thalassaemic plasma as it does in normal plasma. Similarly, the differentiation of normal erythroblasts is not affected by 

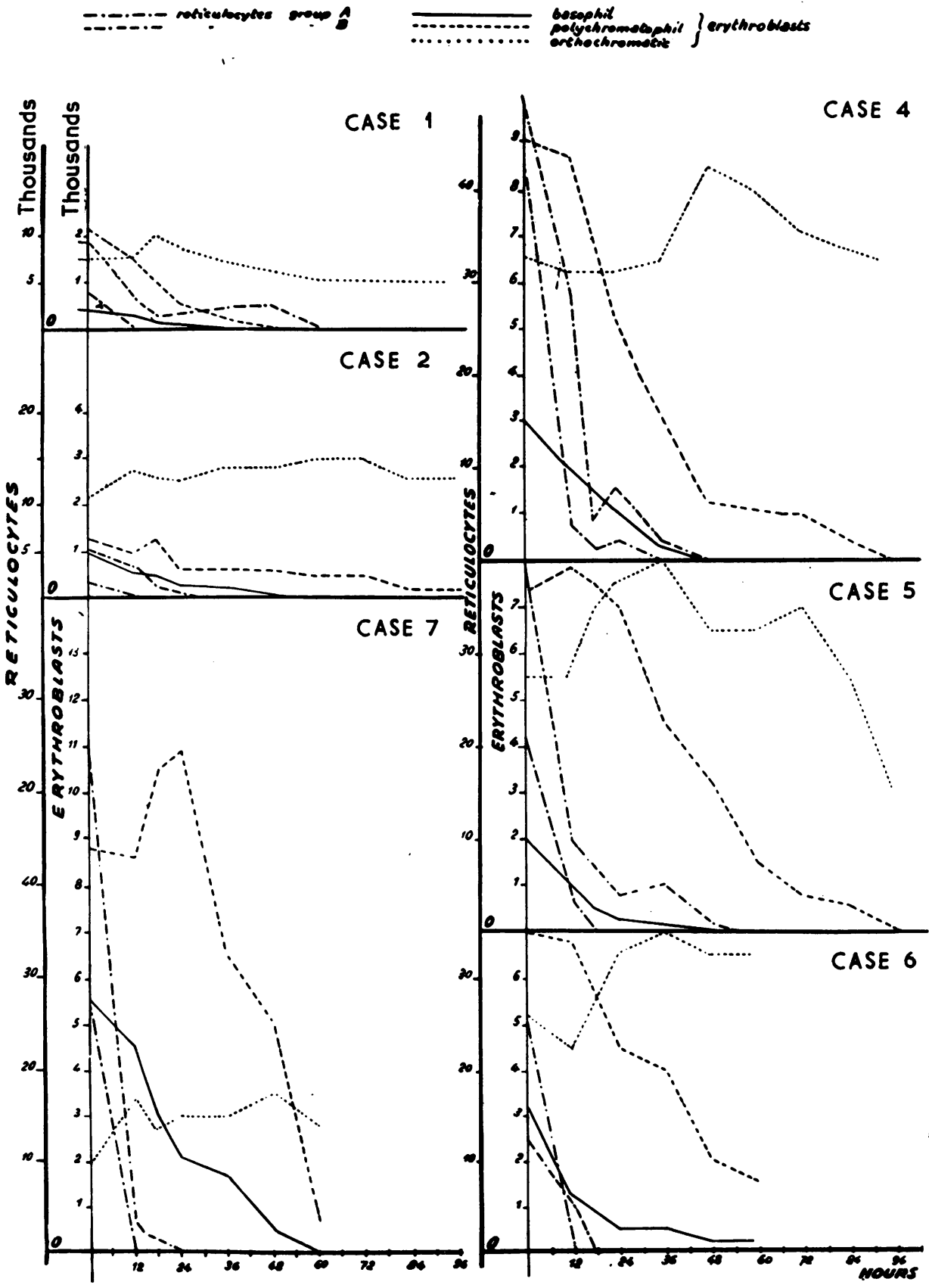

FIG. 2.-Differentiation of thalassaemia major. 
thalassaemic plasma. It can thus be inferred that the abnormal proliferation and differentiation of the erythroblasts in thalassaemia are inherent characteristics, not affected by the type of plasma in which the cells are cultured.

\section{Discussion}

Compensatory Erythropoiesis in Thalassaemia.-The considerable increase in the rate of erythropoiesis in thalassaemia is matched by an increase in the rate of erythrocyte destruction so that a proportional relationship exists between the two phenomena. Both are less intense in thalassaemia minima than in the minor and major forms. Thalassaemia minima may in fact be accompanied by polycythaemia, in spite of the existence of increased haemolysis which, though not so marked as in the major and minor forms, may bring about erythrocyte destruction at twice the normal rate (Astaldi, Tolentino, Sacchetti, and Nonato, 1951).

The erythroblastosis of the bone marrow is thus considered to be a result of the increased haemolysis rather than a primary ana-dysplastic erythroblastosis. In thalassaemia minima, where the destruction of erythrocytes is not very excessive, compensation is complete, or there may be over-compensation and polycythaemia. In the minor and major forms, on the other hand, the rate of destruction is so great that the losses cannot be made good.

Mechanism of Haemolysis. - Of the three mechanisms by which erythrocytes may be removed from the circulation, fragmentation, lysis, and phagocytosis, the first seems to be the most important in thalassaemia. To it must be attributed the bizarre forms of erythrocytes and the smallest $(2-3 \mu)$ microcytes. This phenomenon does not seem to be due to an increased erythrocyte mechanical fragility, for

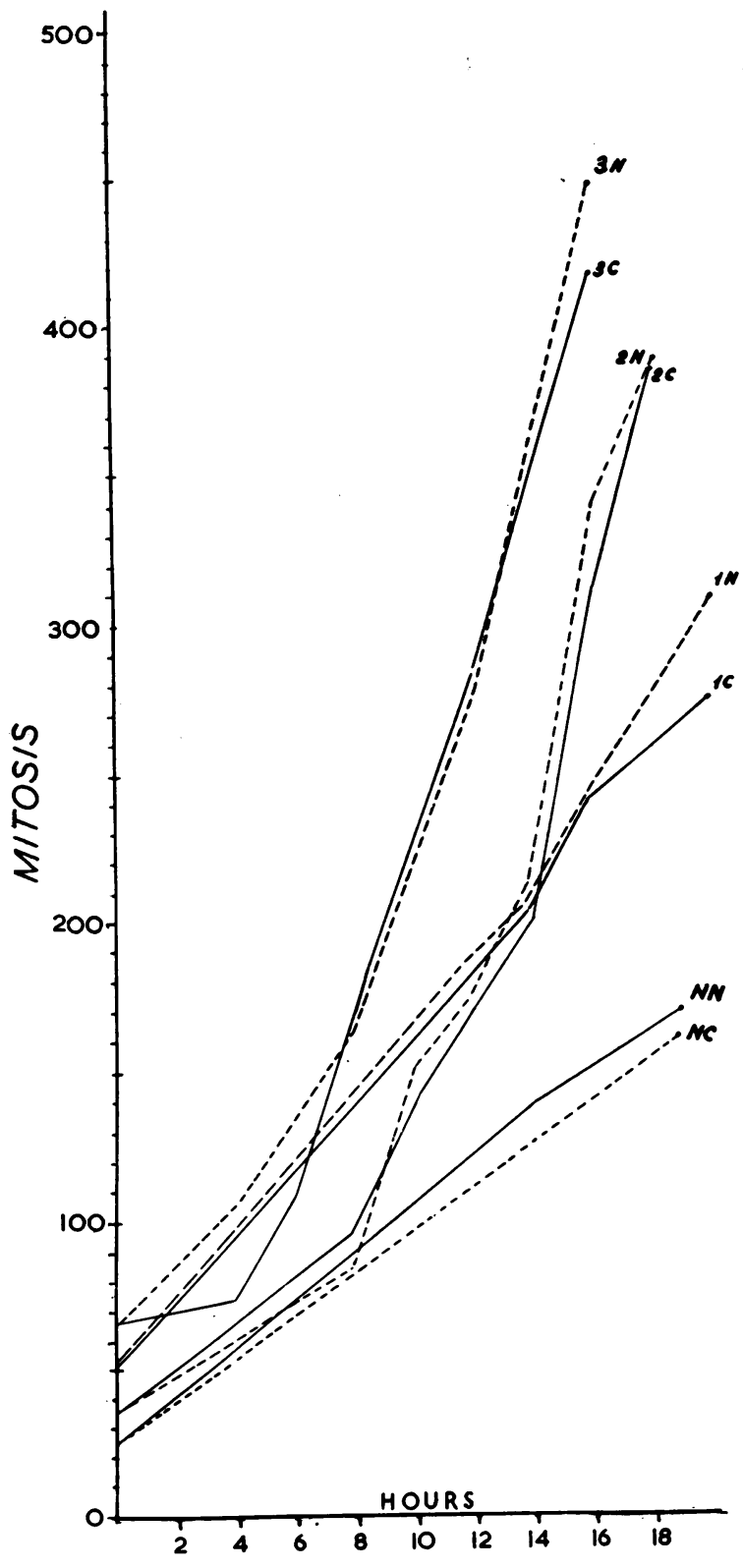

Fig. 3.-Shows " crossed plasma" proliferation. 1C, $2 \mathrm{C}, 3 \mathrm{C}=$ thalassaemia erythroblasts in autoplasma. $\quad 1 \mathrm{~N}, 2 \mathrm{~N}, 3 \mathrm{~N}=$ thalassaemia erythroblasts in normal plasma. $\mathrm{NN}=$ normal erythroblasts in autoplasma. $\mathrm{NC}=$ normal erythroblasts in thalassaemia plasma. 
tests in vitro have shown a higher resistance to trauma (Tolentino, 1950, 1951). The process of spontaneous fragmentation seems rather to be due to intrinsic causes, and may even be seen to be taking place in young forms (reticulocytes).

The modifications produced as a result of splenectomy are not such as support the existence in thalassaemia of a hypersplenic condition. On the contrary, the evidence is in favour of hyposplenism. Similarly, the evidence is against the hypothesis that an abnormality in the plasma determines the increased haemolysis. In fact, the results all point to the conclusion that this increased rate of blood destruction is due to the abnormality of the erythron itself, this notwithstanding the fact that the medullary erythroblastosis is not the morphological expression of such an abnormality but only the consequence of the increased peripheral destruction.

\section{Summary}

The pathogenesis of thalassaemia can thus be summarized as follows. The disease is due to a gene mutation of variable expressivity (Gatto, 1947 ; Silvestroni, 1947) which affects the erythron and causes the formation of morphologically and biologically abnormal erythrocytes. The biological anomaly determines a shorter average life span, resulting in a permanent increase in the rate of haemolysis. A chronic stimulation of the bone marrow with secondary hyperplasia results. The hyperplasia depends on an increased proliferation of basophilic erythroblasts and probably also on a direct differentiation of the histoid matrix.

In thalassaemia minima (the carrier condition) the increase in erythropoiesis makes up for the increased rate of haemolysis and anaemia is avoided. The mating of two thalassaemia minima carriers can result in the birth of homozygous children and these will be afflicted with thalassaemia major. The summation of the effects of two mutant genes prevents the possibility of erythropoiesis balancing haemolysis, for the life span of the erythrocyte is then shorter than in the minima form. The compensatory erythropoiesis is inadequate, even after maximal proliferation of the basophilic erythroblasts and an increase in histoid erythropoiesis to the extent of filling up all the medullary cavities with active erythroblastic tissue, enlarging the diplöe, and invading areas and organs which have as a rule no erythropoietic activity. In such cases the anaemia becomes progressively worse, and periods of bone marrow exhaustion may result in a slowing down of erythroblastic differentiation. The enucleation of orthochromatic erythroblasts is particularly affected and marked erythroblastaemia is evidence of this.

In thalassaemia minor, which is, like thalassaemia minima, a heterozygous condition, the picture is similar to that of thalassaemia major. It is less severe, however, and is not incompatible with life.

REFERENCES

Astaldi, G., and Mauri, C. (1949). Haematologica, 33, 583. (1950). Sang, 21, 378.

and Tolentino, P. (1949). Journal of Clinical Pathology, 2, 217. and Sacchetti, C. (1951a.) Rev. Hémat., 6, 157. (1951b). Helv. paediat. Acta, 6, 50 .

(1951c). La Talassemia. (Bibl. Haemat.) Tipografia del Libro. Pavia. - and Nonato, M. G. (1951). Acta haemat., Basel, 5, 270.

Gatto, I. (1947). Riv. Pediatr. Sicil., $2,20$.

Silvestroni, E. (1947). Policlinico, Sez. prat., 54, 1147.

Tolentino, P. (1950). Boll. Soc. ital. Biol. sper., 26, 251.

(1951). Nature, Lond., 167, 905. 